\title{
Effects of the Nanoparticle-Based Vaccine, SEL-068, on Nicotine Discrimination in Squirrel Monkeys
}

\author{
Rajeev I Desai*,I and Jack Bergman' \\ 'Preclinical Pharmacology Laboratory, Department of Psychiatry, McLean Hospital/Harvard Medical School, Belmont, MA, USA
}

\begin{abstract}
A key feature of addiction to nicotine likely resides in its ability to produce subjective effects that, in turn, may be reflected in its discriminative-stimulus properties. Vaccination against such effects of nicotine offers an intriguing therapeutic approach for smoking cessation, but a reliably effective and immunologically safe vaccine remains to be identified. Here we report on the ability of SEL-068, a nanoparticle-based vaccine that targets nicotine, to modify the discriminative-stimulus effects of nicotine in a primate species. Results indicate that squirrel monkeys vaccinated with SEL-068 failed to acquire $0.1 \mathrm{mg} / \mathrm{kg}$ nicotine discrimination but readily learned to discriminate $0.00 \mathrm{I} \mathrm{mg} / \mathrm{kg}$ of the nicotinic full agonist (+)-epibatidine ((+)-EPI). After (+)-EPI training, doses of nicotine $\geqslant 0.32 \mathrm{mg} / \mathrm{kg}$, which produced behaviorally adverse actions, still failed to substitute for the (+)-EPI training stimulus in immunized monkeys, whereas (+)-EPI and the partial agonist varenicline engendered, respectively, complete and partial substitution in all monkeys with potency comparable to their potency in non-immunized subjects. In other subjects, nicotine was trained as a discriminative-stimulus and then replaced by $(+)$-EPI. Subsequent vaccination with SEL-068 led to a threefold and long-lasting ( $>30$ weeks) decrease in the potency of nicotine but not (+)-EPI or varenicline. Collectively, our results show that SEL-068 can block the development of nicotine discrimination and attenuate nicotine's effects in nicotine-experienced monkeys without altering the discriminative-stimulus properties of other nicotinic drugs. The difference in the vaccine's effects in naive and nicotine-experienced subjects provides important insight into the conditions under which immunotherapy may be effective in combating nicotine addiction.

Neuropsychopharmacology (2015) 40, 2207-22I6; doi: I0.1038/npp.2015.64; published online 15 April 2015
\end{abstract}

\section{INTRODUCTION}

Tobacco-related diseases kill approximately 480000 people each year in the United States alone and are a leading cause of preventable disease and premature death (US Department of Health and Human Services (USDHHS), 2014). Nevertheless, recent reports estimate that $18 \%$ (42 million) of Americans aged $\geqslant 12$ years still use tobacco products (US Department of Health and Human Services (USDHHS), 2014). The persistence of tobacco use in the face of overwhelming evidence of its harm is thought to reflect the highly addictive nature of tobacco constituents. Several smoking cessation and prevention strategies, including behavioral and pharmacological interventions (eg, nicotine replacement therapy, bupropion, and varenicline), are designed to primarily target nicotine's addictive properties. Unfortunately, these treatment approaches are characterized by low rates of success and disappointingly high rates of relapse in individuals who want to quit (Polosa and Benowitz, 2011). Thus there is a considerable need for new smoking cessation

\footnotetext{
*Correspondence: Dr RI Desai, Preclinical Pharmacology Laboratory, Department of Psychiatry, McLean Hospital/Harvard Medical School, I 5 Mill Street, Belmont, MA 02478, USA, Tel: + I 6178553303 , E-mail: rdesai@mclean.harvard.edu

Received 14 December 2014; revised 26 February 2015; accepted 2 March 2015; accepted article preview online 6 March 2015
}

treatment strategies to effectively and safely reduce tobacco consumption.

Immunotherapeutic strategies (eg, nicotine vaccines) have been prominently forwarded as alternative or adjunct treatment approaches to the use of pharmacological agents for smoking cessation (eg, Hartmann-Boyce et al, 2012; Raupach et al, 2012; Pentel and LeSage, 2014). Effective vaccination against nicotine should promote an immune response characterized by elevated levels of antibodies that selectively identify, bind, and sequester nicotine molecules in systemic circulation outside the CNS, thereby precluding or attenuating addiction-related effects (eg, Raupach et al, 2012). However, the development of an effective vaccine against nicotine has been challenging. Several different formulations of first-generation nicotine-targeting vaccines-entailing protein conjugate immunogens linked to nicotine or conjugated to a carrier protein and mixed with an adjuvant-have failed in clinical trials, despite preclinical data showing that they can: (a) alter nicotine's pharmacokinetic properties; (b) interfere with nicotine's abuse-related neurochemical and behavioral actions; and (c) lessen nicotine withdrawal symptoms (Carrera et al, 2004; Lindblom et al, 2002, 2005; de Villiers et al, 2002; LeSage et al, 2006; Hartmann-Boyce et al, 2012; Raupach et al, 2012; Pentel and LeSage, 2014). Yet, only modest increases in quit rates have been observed in smokers with high levels of antinicotine antibodies (eg, Hartmann-Boyce et al, 2012). 
Although the reasons for such failures are not certain, a frequently voiced concern has involved the inability of firstgeneration antinicotine vaccines to consistently produce a robust immune response (ie, high antibody levels) in all immunized subjects, perhaps attributable to their limited immunogenicity, affinity, and selectivity in man (HartmannBoyce et al, 2012; Pentel and LeSage, 2014).

Recent advances in vaccine development have led to innovative second-generation nicotine-targeting vaccines that are expected to produce a more consistent immune response (and, presumably, improved efficacy) and lesser potentially adverse effects (Pittet et al, 2012; Kishimoto et al, 2012; Fraser et al, 2014; Pentel and LeSage, 2014). Along these lines, Kishimoto and co-workers have described the development of SEL-068, a novel nanoparticle-based vaccine ( $t \mathrm{SVP}$ ) with key components recognized by the immune system to mount a strong response to a pathogen; these include a biodegradable and biocompatible polymer matrix, a synthetic TLR agonist, a novel universal T-cell helper peptide, and nicotine covalently conjugated to the nanoparticle surface (B-cell antigen; Fraser et al, 2014; further details regarding this vaccine are provided at http://www.selectabio. com). Interestingly, preclinical studies have shown SEL-068 to robustly and dose-dependently induce high-affinity antinicotine antibody titers in both mice and nonhuman primates (Pittet et al, 2012; Kishimoto et al, 2012; Fraser et al, 2014). On the basis of such early observations, the development of nanoparticle-based vaccines that target nicotine has been proposed as a promising immunotherapeutic strategy for preventing tobacco consumption (Pittet et al, 2012; Kishimoto et al, 2012; Fraser et al, 2014; Pentel and LeSage, 2014).

Notwithstanding advances in the effort to develop nanoparticle-based vaccines (Boston Business Journal, 17 June 2014; http://www.bizjournals.com/boston/blog/bioflash/ 2014/06/selecta-gets-8-1m-nih-grant-for-more-trials-of.html), there has been scant preclinical information on the effectiveness of such synthetic nanoparticle-based nicotine vaccines in preventing behavioral effects of nicotine that may be involved in initiating or relapsing to smoking behavior. The present behavioral studies were conducted to address this question by determining how a treatment regimen of SEL-068 that can be expected to produce a robust immune response also modifies nicotine's discriminative-stimulus effects, which have been related to its subjective effects in man (eg, Smith and Stolerman, 2009; Kamien et al, 1993). Standard drug discrimination procedures (eg, Smith and Stolerman, 2009; Jutkiewicz et al, 2011; Cunningham et al, 2012; Perkins, 2009; Desai and Bergman, 2014) were employed to determine whether vaccination with SEL-068 might prevent, or when already established, attenuate nicotine's discriminativestimulus effects in nonhuman primates.

\section{MATERIALS AND METHODS}

\section{Subjects}

Eight experimentally naive adult male squirrel monkeys (Saimiri sciureus; University of Texas, MD Anderson Center, Bastrop, Texas, USA), weighing 700-900 g, served as subjects. The housing and feeding protocols for all subjects were comparable to those employed previously in our laboratory
(Desai and Bergman, 2014). Behavioral experiments were conducted daily (Monday-Friday) between 0800 and 1800 hours under protocols that were approved by the Institutional Animal Care and Use Committee at McLean Hospital. Subjects were maintained in the McLean Hospital Animal Care Facility (licensed by the US Department of Agriculture) in accordance with guidelines provided by the Committee on Care and Use of Laboratory Animals of the Institute of Laboratory Animals Resources, Commission on Life Sciences, National Research Council (2011).

\section{Apparatus}

During experimental sessions, monkeys sat in customized Plexiglas chairs (Kelleher and Morse, 1968; Desai and Bergman, 2014) that were enclosed in ventilated, sound-attenuating chambers; white noise was present at all times to mask extraneous sounds. While seated, monkeys faced a panel containing two sets of colored stimulus lights and two response levers that were comfortably within the subject's reach, one below each set of stimulus lights. Each lever-press with a force $>0.2 \mathrm{~N}$ produced an audible click and was recorded as a response. Prior to each behavioral session, a shaved portion of the monkey's tail was secured under brass electrodes by a small stock for the delivery of brief, lowintensity stimuli ( $3 \mathrm{~mA}$ for $200 \mathrm{msec}$; current delivery). These parameters were chosen on the basis of previous research in our laboratory (Tidey and Bergman, 1998; Desai and Bergman, 2014). The shaved area was coated with electrode paste to ensure a low-resistance electrical contact between electrodes and tail. Experimental contingencies and data collection were controlled and recorded in an adjoining room via Med Associates interfacing equipment and operating software (MED-PC, MedState Notation, Med Associates, St Albans, VT, USA).

\section{Drug Discrimination}

Experimental groups. Studies were conducted to evaluate the effects of immunization with SEL-068 on nicotine discrimination and to determine the dose-related effects of nicotine, (+)-epibatidine, and varenicline under differing experimental conditions. A detailed timeline of experimental protocols in the present study is shown in Figure 1. Briefly, in one group of four monkeys (Group A), dose-response functions for each drug were determined, first, after subjects reliably discriminated nicotine and before vaccination with SEL-068 (2 mg per injection, s.c.; see Figure 1 for further details) and, subsequently, after subjects reliably discriminated (+)-epibatidine and after vaccination with SEL-068. In the second group of four monkeys (Group B), dose-response functions for each drug were determined after the acquisition of (+)-epibatidine discrimination, which followed both treatment with SEL-068 (2 mg per injection, s.c.; see Figure 1 for further details) and discrimination training with nicotine.

Experimental training. The subjects were divided into two groups ( $n=4$ /group) for the present experiments. Initially, subjects were trained to press each response lever under a 10response fixed-ratio (FR10) schedule of stimulus termination (Desai and Bergman, 2014). Under this schedule, current delivery was scheduled to be delivered to the tail every $10 \mathrm{~s}$ 

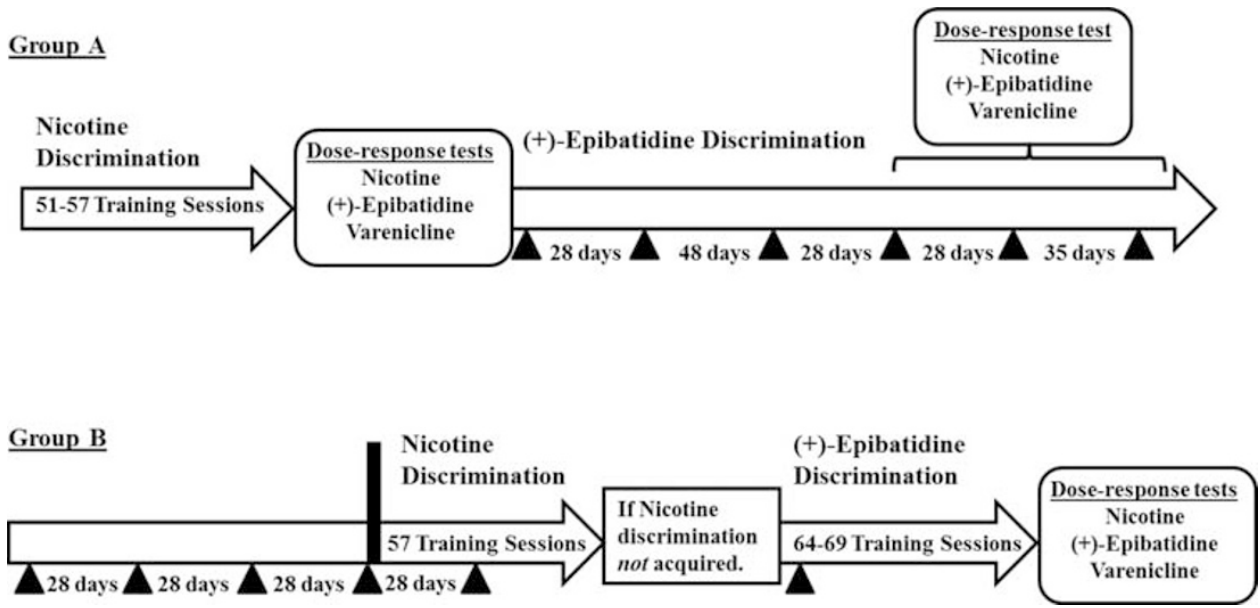

Figure I Timeline of experimental protocols in two different groups of squirrel monkeys. Group A: Subjects were trained to identify 0.1 mg $/ \mathrm{kg}$ nicotine as a discriminative-stimulus using the procedures described in Methods section. Once subjects met criteria for successful nicotine discrimination, the dose-related effects of nicotine, (+)-epibatidine, and varenicline were determined. Subsequently, $0.00 \mathrm{l} \mathrm{mg} / \mathrm{kg}(+)$-epibatidine replaced $0.1 \mathrm{mg} / \mathrm{kg}$ nicotine as the training drug, and the vaccination protocol similar to the one used in Group B (see below) was initiated in all subjects. After criteria for discrimination performance were again met, test sessions with nicotine were conducted once a week for up to 30 weeks to determine whether the previously recognized training dose of nicotine $(0.1 \mathrm{mg} / \mathrm{kg})$ substituted for (+)-epibatidine's discriminative-stimulus effects. After $0.1 \mathrm{mg} / \mathrm{kg}$ nicotine failed to serve as a discriminative-stimulus, doseeffect functions for the ability of nicotine, (+)-epibatidine, and varenicline to substitute for (+)-epibatidine were determined in all subjects. Group B: All subjects were treated with subcutaneous (s.c.) injections of $2 \mathrm{mg}$ of SEL-068 on four separate occasions (indicated by $\mathbf{\Delta}$ ). During this time, all subjects were trained to respond under the FRIO schedule of stimulus termination (without exposure to nicotine or other drugs) in preparation for later nicotine-discrimination training. Next, the acquisition of nicotine as a discriminative-stimulus was studied in each subject as described in Methods section. All subjects received a fifth s. c. injection of $2 \mathrm{mg}$ of SEL-068 once both levers were available (ie, after 24 sessions). If acquisition of $0.18 \mathrm{mg} / \mathrm{kg}$ nicotine as a discriminative-stimulus was not evident within 57 behavioral training sessions (ie, 33 training sessions: 24 single lever sessions +33 sessions after both levers are available, the time frame that was established for successful acquisition of nicotine discrimination in Group A), $0.0018 \mathrm{mg} / \mathrm{kg}(+)$-epibatidine was substituted for nicotine as the training drug. A sixth injection of $2 \mathrm{mg}$ of SEL-068 was administered to all subjects before the start of training with $0.00 \mathrm{l} 8 \mathrm{mg} / \mathrm{kg}(+)$-epibatidine. Subsequently, the protocol for discrimination training was repeated with $(+)$-epibatidine as the training drug. Once subjects met criteria for testing, studies with nicotine, $(+)$-epibatidine, and varenicline were conducted.

when stimulus lights above the levers were illuminated. However, every tenth lever press or the fourth current delivery turned off the illuminated stimulus lights for a 50-s timeout period (TO $50 \mathrm{~s}$ ). After initial training, subjects typically completed the response requirement within several seconds after the stimulus lights were first illuminated, thus turning them off and beginning the TO $50 \mathrm{~s}$ without the delivery of current. Once stable performance was established on both response levers under the FR10;TO50 s schedule, drug discrimination training was initiated. Subjects underwent two phases of training to discriminate intramuscular (i.m.) injections of saline from i.m. injections of nicotine or, as a structurally distinct but pharmacologically similar substitute, (+)-epibatidine. (+)-Epibatidine, a potent and selective $\alpha 4 \beta 2$ nicotinic receptor agonist, previously has been shown to produce discriminative-stimulus effects that are comparable to those of nicotine (Damaj et al, 1994; Desai and Bergman, 2014). During initial training (24 sessions), only one of the two levers, the injection-associated lever for that day, was accessible to the subject, and the type of injection (drug (D) or saline (S)) generally followed a doublealternation schedule (SS-DD-SS-DD). Thus, on days when drug $(0.18 \mathrm{mg} / \mathrm{kg}$ nicotine or $0.0018 \mathrm{mg} / \mathrm{kg}(+)$-epibatidine $)$ or saline was injected before the session, the completion of 10 consecutive responses on the accessible lever was reinforced. During subsequent training (Session 25 onwards), experimental contingencies were identical to those during the initial 24 behavioral sessions, with the exception that both response levers were accessible to the subject in all sessions.
The assignment of drug- and saline-associated levers was counterbalanced across monkeys. During training session in which both levers were available, a response on the incorrect lever (for that day's injection) reset the FR response requirement on the correct lever to 10; this program feature discouraged indiscriminate responding on both levers. When stable discrimination performance was achieved across daily training sessions (ie, after four consecutive sessions in which $>90 \%$ of responses were on the injection-associated lever), the training dose of nicotine or (+)-epibatidine was reduced to 0.1 or $0.001 \mathrm{mg} / \mathrm{kg}$ i.m., respectively, and a double alternation schedule of presession injections was continued for all subsequent training sessions (eg, for nicotine: saline-nicotine-nicotine-saline-etc; for (+)-epibatidine: saline-(+)-epibatidine-(+)-epibatidine-saline-etc). During this terminal portion of discrimination training and thereafter, all training sessions consisted of 10 presentations of the FR10;TO 50-s schedule, preceded by a 10-min TO period during which saline or drug (nicotine or $(+)$-epibatidine) could be administered. Training sessions continued until all subjects met criteria for stability, ie, $>90 \%$ of responses on the injection-associated lever in the first FR and overall during both the preceding training session and four of the last five training sessions. Thereafter, test sessions were conducted when subjects met the above criteria for stability in two consecutive alternating (S-D or D-S) training sessions. Injections of a test drug or saline were administered at the beginning of the 10-min TO periods preceding test sessions. Test sessions were identical to training sessions, with two 
exceptions: first, 10 consecutive responses on either lever turned off all stimulus lights and programmed consequences and, second, the current generator was turned off to preclude responding elicited by electric stimuli during testing. Studies were conducted as described above under experimental groups as shown in Figure 1.

\section{Drugs and Dosing Procedures}

(-)-Nicotine ([- ]-1-methyl-2-[3-pyridyl]pyrrolidine) hydrogen tartrate was obtained from Sigma-Aldrich (St Louis, MO). (+)-Epibatidine [(2 R)-2-(6-chloro-3-pyridinyl)-7azabicyclo[2.2.1] heptane monohydrochloride] was obtained from the National Institute on Drug Abuse (Bethesda, MD). Varenicline (6,7,8,9-tetrahydro-6,10-methano-6H-pyrazino [2,3-h][3]benzazepine) was generously donated by Dr Hans Rollema from Pfizer Global Research and Development (Department of Neuroscience Biology, Eastern Point Road, Groton, CT 06340, USA; present address: Rollema Biomedical Consulting, Mystic, CT, USA). Doses of nicotinic agonists and drug preparation procedures were based on previous studies in our laboratory (Desai and Bergman, 2014). All drugs were dissolved in $0.9 \%$ saline and were administered by i.m. injection. The $\mathrm{pH}$ of nicotine and varenicline were adjusted as needed to 7.0 with $0.1 \mathrm{~N}$ sodium hydroxide. A full range of doses of nicotine (0.0032-0.32 or $0.56 \mathrm{mg} / \mathrm{kg}$, i.m.), (+)-epibatidine $(0.000032-0.001 \mathrm{mg} / \mathrm{kg}$, i.m.), and varenicline $(0.01-0.18 \mathrm{mg} / \mathrm{kg}$, i.m.) were studied in Group A and B subjects as described above. Doses of each drug are expressed in terms of the free base. The nanoparticle-based vaccine, SEL-068, was provided by Selecta Biosciences (Watertown, MA, USA). On immunization days, SEL-068 was freshly prepared for the s.c. delivery of a $0.3 \mathrm{ml}$ solution containing $2 \mathrm{mg}$ SEL-068.

\section{Data Analysis}

The percentage of responding that occurred on the drugassociated lever during a session was calculated by dividing the number of responses on that lever by the total responses on both levers. Response rates were calculated for each session by dividing the total number of responses by the duration of the session minus timeout periods. If the mean response rate in a session was $<0.2$ responses/s, data from that session were excluded from further analysis. Mean results for vehicle and each dose of a drug were calculated by averaging data of the four subjects within that group. Substitution profiles for test drugs were defined for individual subjects and for the group of subjects as full $(\geqslant 90 \%$ drug-associated lever responding), intermediate (31-89\% drug-associated lever responding), and absent ( $<30 \%$ drugassociated lever responding) (Desai and Bergman, 2014).

When appropriate, a two-way repeated measures analysis of variance (ANOVA) with treatment and time as factors followed by Dunnett's $t$-test or a paired $t$-test was used to evaluate statistical significance of averaged data for percentage of drug-associated lever responding and response rates (defined at the 95\% level of confidence; $P<0.05$; GraphPad Prism 5.02, GraphPad Software, La Jolla, CA, USA). All results of statistical analyses are shown in the figure legends.

\section{RESULTS}

\section{Nicotine Discrimination Before and After Immunization with SEL-068}

The acquisition of discriminative-stimulus control by $0.1 \mathrm{mg} / \mathrm{kg}$ nicotine prior to vaccination with SEL-068 (Group A) is shown in Figure 2 (left panels). Discriminative-stimulus control by $0.18 \mathrm{mg} / \mathrm{kg}$ nicotine developed quickly, evident in a clear separation in the distribution of behavior after the injection of $0.18 \mathrm{mg} / \mathrm{kg}$ nicotine ( $\approx 50 \%$ drug-lever responding) and vehicle ( $\approx 20 \%$ drug-lever responding) within the first several discrimination training sessions once both response levers were available (ie, after 24 sessions; Figure 2, top left panel). The ability to discriminate $0.18 \mathrm{mg} / \mathrm{kg}$ nicotine from injections of vehicle gradually increased across subsequent training sessions, with all four subjects responding exclusively on the injection-appropriate lever after approximately 47 training session $(0.18 \mathrm{mg} / \mathrm{kg}$ nicotine $\geqslant 90 \%$ drug-lever responding; vehicle $\leqslant 10 \%$ drug-lever responding; Figure 2, top left panel). Once individual subjects met criteria for stability (see Methods section), the training dose of nicotine was reduced to $0.1 \mathrm{mg} / \mathrm{kg}$. All monkeys again met criteria for nicotine discrimination within the next 10 training sessions (during training sessions 51 to 57; Figure 2, top left panel). Rates of responding for all subjects increased steadily over the course of discrimination training, which is reflected in the mean data for the group of monkeys (average of sessions $25-35$ response rate: nicotine $=1.82 \pm 0.16$, saline $=1.83 \pm$ 0.16 ; average of sessions $47-57$ response rate: nicotine $3.14 \pm 0.13$, saline $=2.76 \pm 0.13$; Figure 2, bottom left panel) Mean rates of responding after injections of nicotine did not differ consistently from mean response rates after vehicle injection over the course of nicotine-discrimination training (Figure 2, bottom left panel).

The effects of nicotine-discrimination training in subjects after vaccination with SEL-068 (Group B) are shown in Figure 2 (right panels). These data indicate that vaccinated subjects, unlike non-vaccinated subjects (Group A), failed to acquire the discrimination of $0.18 \mathrm{mg} / \mathrm{kg}$ nicotine from vehicle. Thus, injection-appropriate responding in vaccinated subjects remained at approximately chance levels over the course of approximately the same number of training sessions as in Group A (56-57; compare Figure 2 top left and right panels). No differences in mean response rates after injections of nicotine or vehicle were observed between the two groups (Figure 2, bottom right panel).

\section{(+)-Epibatidine Discrimination After Vaccination with SEL-068}

SEL-068-treated subjects (Group B) that did not learn to discriminate $0.18 \mathrm{mg} / \mathrm{kg}$ nicotine from vehicle developed reliable and robust (+)-epibatidine discrimination (Figure 3, top panel). Although approximately a dozen more sessions were required to fully meet criteria for discrimination of the training stimulus in experiments with (+)-epibatidine than nicotine (64-69 vs 51-57), the overall patterns of acquisition of discriminative-stimulus control by (+)-epibatidine (Group B) and nicotine (Group A) were similar. As with nicotine, stimulus control following injections of the initial training dose of (+)-epibatidine developed relatively quickly once both levers were made available (in approximately 30 training sessions) 
Group A
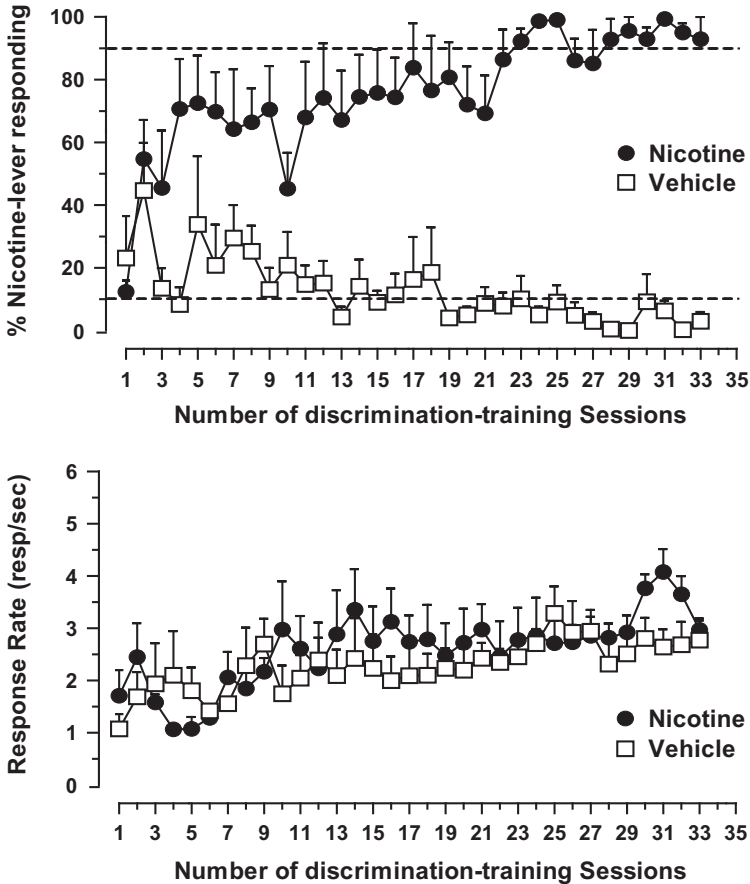

Group B
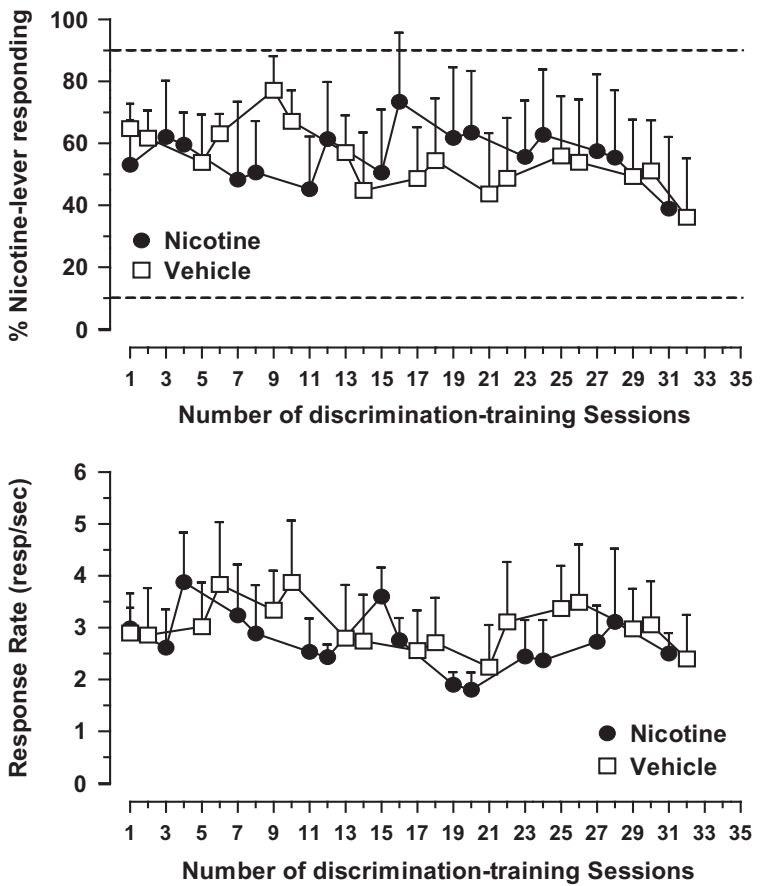

Figure 2 Group A, left top and bottom panels: Acquisition of stimulus control in a group of four squirrel monkeys trained to discriminate $0.1 \mathrm{mg} / \mathrm{kg}$ nicotine from vehicle (saline; Group A). Abscissae: number of training session once single lever training was completed $(\mathrm{I}-24)$ and both levers were available (session 25 onward; ie, 33 training sessions: 24 single lever sessions +33 sessions after both levers are available). Ordinates: effect shown as the percentage of lever-press responses occurring on the nicotine-lever (top) and response rates (bottom) following injections of nicotine or vehicle. The horizontal dashed line at $90 \%$ nicotine-lever responding (top left panel) indicates the criterion for evidence of discriminative-stimulus control. Each point represents the mean value and the standard error of the mean (SEM). The dose of nicotine (i.m.) was reduced progressively from 0.18 to $0.1 \mathrm{mg} / \mathrm{kg}$ in individual subjects when they met criteria (see Methods section). ANOVA confirmed significant main effects of drug treatment $\left(F_{1,192}=49.29 ; P<0.05\right)$, time $\left(F_{32,192}=1.64 ; P<0.05\right)$, and a treatmentby-time interaction $\left(F_{32,192}=4.92 ; P<0.05\right)$ on the percentage of nicotine-appropriate responding. No significant effects on response rates were observed after injections of nicotine or vehicle (treatment: $F_{1,192}=0.38 ; P>0.05$; treatment-by-time interaction: $F_{32,192}=1.28 ; P>0.05$; bottom panel). A significant effect of time on response rates was obtained $\left(F_{32.192}=3.86 ; P<0.05\right)$, most likely reflecting some increases in the overall rates of responding in all subjects over the course of discrimination training. Group B, right top and bottom panels. Lack of stimulus control in a group of four squirrel monkeys that were treated with SEL-068 and subsequently trained to discriminate $0.18 \mathrm{mg} / \mathrm{kg}$ nicotine from vehicle (saline; Group B). See left top and bottom panels of Group A for all other details. ANOVA confirmed no significant effects of drug treatment ( $\left.F s_{1,90} \geqslant 0.0002 ; P_{S}>0.05\right)$, time (Fs $\left.15,90 \geqslant 0.61 ; P_{S}>0.05\right)$, or a treatment-by-time interaction $\left(\mathrm{Fs}_{15,90} \geqslant 0.7 ; \mathrm{PS}_{\mathrm{S}}>0.05\right)$ on the percentage of nicotine-appropriate responding and response rates during $0.18 \mathrm{mg} / \mathrm{kg}$ nicotine discrimination training.

and nearly exclusive selection of the injection-appropriate lever was observed in all four subjects after approximately 58 training sessions (compare Figures 2 and 3). After the training dose was reduced to $0.001 \mathrm{mg} / \mathrm{kg}$ (i.m.) (+)-epibatidine, all subjects were able to consistently discriminate between (+)-epibatidine and vehicle within an additional 6-10 sessions, ie, after a total of approximately 64-69 training sessions (Figure 3, top panel).

During the acquisition of nicotine and (+)-epibatidine discrimination, mean response rates after injections of nicotine and (+)-epibatidine were not significantly different (ie, approximately 2.5-3 responses/s, respectively; Figure 3, bottom panel). As with nicotine, mean response rates after injections of the training dose of (+)-epibatidine or vehicle were not significantly different $(3.79 \pm 1.72$ vs $4.45 \pm 0.65$; Figure 3, bottom panel), albeit response rates after (+)-epibatidine appeared somewhat lower than after vehicle (Figure 3, bottom panel).

\section{Loss of Nicotine Discrimination After Immunization with SEL-068}

The effects of periodic substitution tests conducted with $0.1 \mathrm{mg} / \mathrm{kg}$ of nicotine in all Group A subjects during treatment with SEL-068 over the course of 30 weeks are shown in Figure 4. Results show that injections of (+)-epibatidine $(0.001 \mathrm{mg} / \mathrm{kg})$ reliably maintained discriminativestimulus control in all subjects throughout these studies (Figure 4, top panel) and that $0.1 \mathrm{mg} / \mathrm{kg}$ of nicotine fully substituted for (+)-epibatidine through the first 7 weeks of the regimen. However, approximately 7 weeks after SEL-068 treatment began (ie, after two injections of SEL-068), the mean percentage of drug-lever responding decreased slightly below criterion levels for complete substitution to approximately $80 \%$ (Figure 4, top panel, filled circles). Nicotine discrimination decreased further to approximately 30-70\% drug-lever responding following two additional injections of SEL-068 between weeks 19 and 22 (Figure 4, top panel). This effect was due to decreases in nicotine discrimination in three of the four subjects. For example, the average value for nicotine discrimination in week 20 was approximately $28 \%$ drug-lever responding, reflecting two subjects at $0 \%$, one subject at $10 \%$, and one subject in which drug-lever responding remained at $100 \%$. Despite some later recovery, nicotine discrimination was substantively decreased in this group of subjects for the remainder of the present 

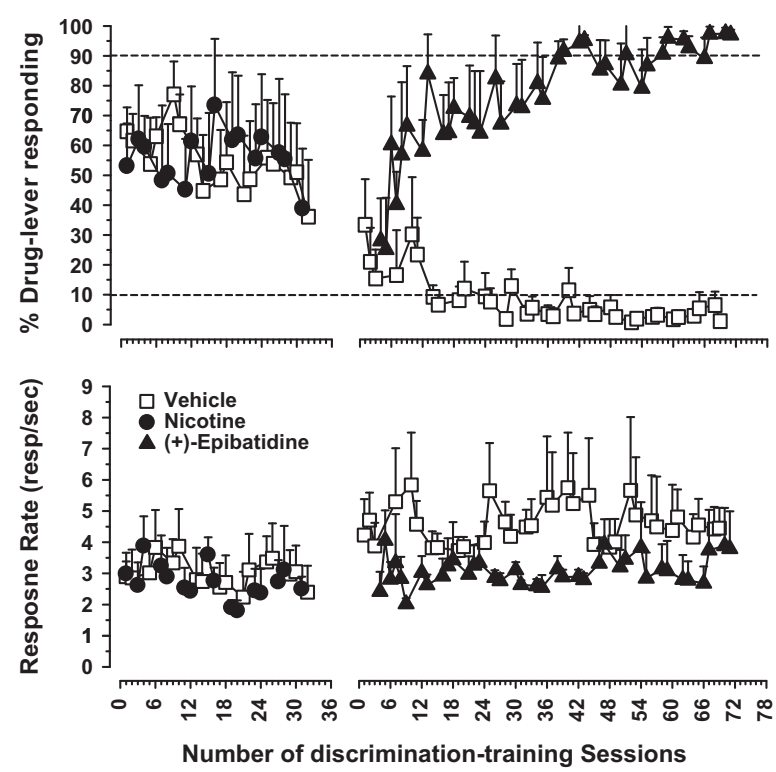

Figure 3 Acquisition of discriminative-stimulus control by $0.001 \mathrm{mg} / \mathrm{kg}$ $(+)$-epibatidine in four immunized squirrel monkeys that failed to learn $0.1 \mathrm{mg} / \mathrm{kg}$ nicotine discrimination (Group B). Data from nicotine discrimination training sessions from Figure 2 (right top and bottom panels) are also included for comparison with (+)-epibatidine discrimination training. Abscissae (top and bottom panels): number of training session once single lever training was completed $(1-24)$ and both levers were available $(25$ sessions onwards). Ordinates: effect shown as the percentage of lever-press responses occurring on the nicotine- or (+)-epibatidine-lever (top panel) and response rates (bottom panel) following injections of nicotine, (+)-epibatidine or vehicle (saline). See Figure 2 for all other details. ANOVA confirmed a significant effect of drug treatment $\left(F_{1,222}=57.56\right.$; $P<0.05)$, a near significant effect of time $\left(F_{37,192}=1.42 ; P<0.05\right)$, and a significant interaction between drug and time $\left(F_{37,222}=5.13 ; P<0.05\right)$ on the percentage of $(+)$-epibatidine-appropriate responding during phase 2 training. Differences in the magnitude of effects among individual subjects precluded statistical significance for the averaged response rate data (drug treatment: $F_{1,222}=3.15 ; P>0.05$; time: $F_{37,222}=0.45 ; P>0.05$; treatmentby-time interaction: $F_{37,222}=0.46 ; P>0.05$ ).

studies (ie, through week 30; Figure 4, top panel). Response rates were not significantly altered by SEL-068 treatment over the course of the 30-week study (Figure 4, bottom panel). Small differences in response rates following injection of vehicle and nicotine or (+)-epibatidine during weeks 1-3 after the first SLE-068 treatment diminished over the course of these experiments (Figure 4, bottom panel).

\section{Substitution Tests with Nicotinic Agonists}

Results from dose-response determinations conducted in non-vaccinated subjects (Group A) following acquisition of $0.1 \mathrm{mg} / \mathrm{kg}$ nicotine discrimination are shown in Figure 5. Prior to treatment with SEL-068, both $0.1 \mathrm{mg} / \mathrm{kg}$ of nicotine and $0.001 \mathrm{mg} / \mathrm{kg}$ of (+)-epibatidine fully substituted for the training dose of nicotine (Figure 5, open symbols, top right and left panels, respectively). The nicotinic partial agonist, varenicline, also substituted for the training dose of nicotine; however, the highest doses of varenicline ( 0.1 and $0.18 \mathrm{mg} / \mathrm{kg}$ ) produced only intermediate levels of responding on the nicotine-associated lever (Figure 5, open symbol, top middle panel). Upon completion of dose-response

determinations, the training drug was switched from $0.1 \mathrm{mg} / \mathrm{kg}$ nicotine to $0.001 \mathrm{mg} / \mathrm{kg}(+)$-epibatidine, and all subjects were treated with SEL-068 following the vaccination protocol described above (see Methods section). Doseresponse redetermination between weeks 19 and 29 (ie, when $0.1 \mathrm{mg} / \mathrm{kg}$ nicotine discrimination was decreased) showed that the substitution profiles of $(+)$-epibatidine and varenicline were unchanged (Figure 5, half-closed symbols, left and middle panels, respectively). However, the doseresponse curve for nicotine was shifted rightward by approximately threefold. Thus subjects no longer identified $0.1 \mathrm{mg} / \mathrm{kg}$ nicotine as a discriminative-stimulus, and full 


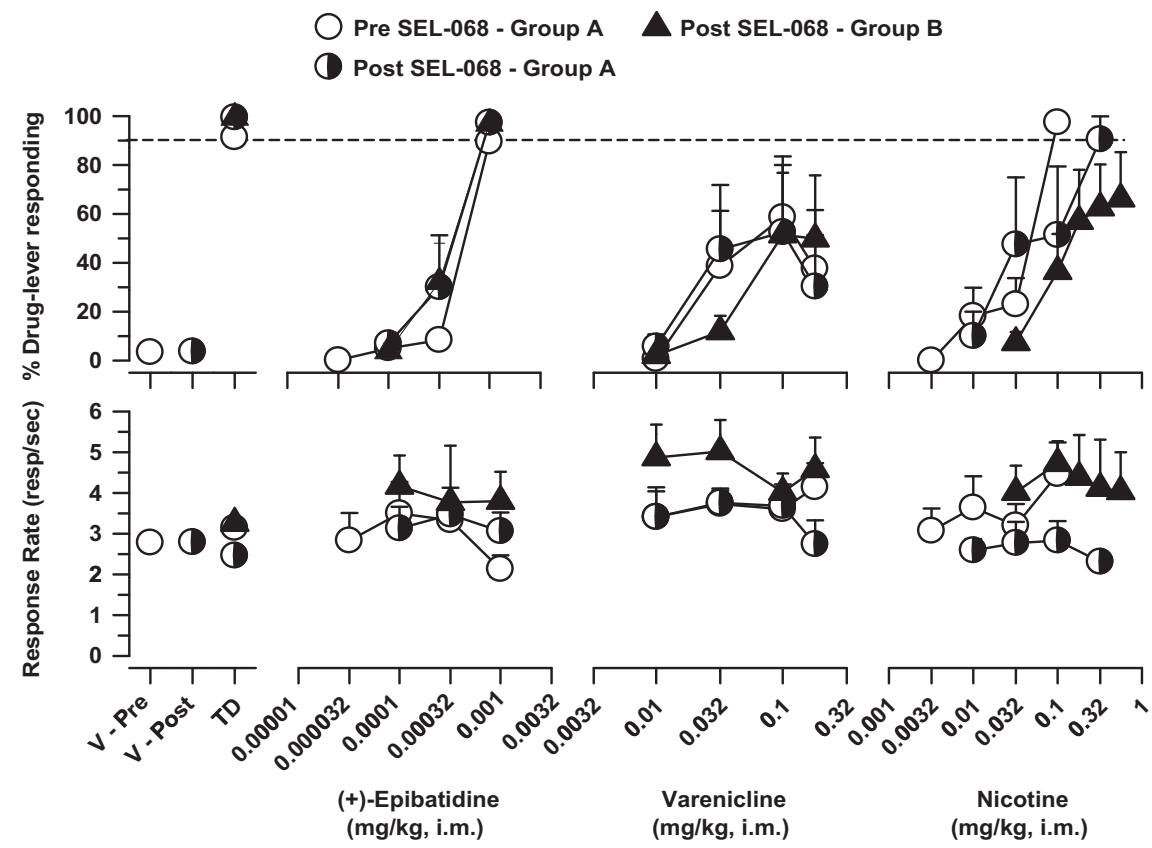

Figure 5 Effects of (+)-epibatidine, varenicline, and nicotine before (open symbols) and after (half-closed symbols) SEL-068 treatment in four squirrel monkeys (Group A) that were initially trained to discriminate $0.1 \mathrm{mg} / \mathrm{kg}$ nicotine from vehicle (ie, before SEL-068 treatment) and subsequently trained to distinguish between $0.001 \mathrm{mg} / \mathrm{kg}(+)$-epibatidine from vehicle (ie, during SEL-068 treatment). Dose-response curves for $(+)$-epibatidine, varenicline, and nicotine determined in squirrel monkeys (Group B) that failed to acquire $0.18 \mathrm{mg} / \mathrm{kg}$ nicotine but not $0.00 \mathrm{I} \mathrm{mg} / \mathrm{kg}(+)$-epibatidine discrimination after immunization with SEL-068 are also shown (fully closed symbols). Ordinates: percentage of drug-lever responding. Abscissae: $V=$ vehicle; TD = training dose; drug dose in $\mathrm{mg} / \mathrm{kg}$ (log scale). Each point represents the mean \pm SEM of data from all four subjects tested at each dose. The horizontal dashed line at $90 \%$ drug-lever responding indicates criteria for complete substitution with a dose of a test drug. During control sessions, the nicotinic agonist training dose (Group A: nicotine or (+)-epibatidine; Group B: (+)-epibatidine) produced $>90$ responding on the drug-associated lever in both Group A and B subjects and injections of saline engendered $\leqslant 3.7 \%$ responding on the drug-associated lever.

substitution for (+)-epibatidine was evident only after the higher dose of $0.32 \mathrm{mg} / \mathrm{kg}$ nicotine in all subjects (Figure 5, half-closed symbols, right panel).

The effects of $(+)$-epibatidine, varenicline, and nicotine were also determined in vaccinated subjects that were trained to discriminate $0.001 \mathrm{mg} / \mathrm{kg}(+)$-epibatidine from vehicle after they failed to acquire $0.18 \mathrm{mg} / \mathrm{kg}$ nicotine discrimination (Group B). Results show that (+)-epibatidine and varenicline produced, respectively, full and intermediate levels of substitution for the $(+)$-epibatidine training stimulus, with potencies and effectiveness that were similar to those in Group A (Figure 5, filled triangles, left and middle panel, respectively). In contrast, the effects of nicotine differed from its effects in unvaccinated Group A subjects (Figure 5, filled triangles, right panel); in two or three monkeys, doses of nicotine up to 0.32 or $0.56 \mathrm{mg} / \mathrm{kg}$ did not fully substitute for the training stimulus. However, as in previous studies, these higher doses of nicotine produced emesis, precluding further increases in dose in these experiments (Figure 5, right panel; Desai and Bergman, 2014).

\section{DISCUSSION}

The present studies were conducted to determine whether SEL-068, a second-generation synthetic nanoparticle-based nicotine-targeting vaccine, could modify the discriminativestimulus effects of nicotine in a primate species. Results indicate that, consistent with previous nicotine discrimination studies in laboratory animals (Desai et al, 2003; Jutkiewicz et al, 2011; Cunningham et al, 2012; see Smith and Stolerman, 2009 for review), non-immunized squirrel monkeys readily learned to discriminate $0.1 \mathrm{mg} / \mathrm{kg}$ nicotine from vehicle. However, monkeys vaccinated with SEL-068 failed to acquire the discrimination of $0.1 \mathrm{mg} / \mathrm{kg}$ nicotine. Moreover, after the training drug was changed to the pharmacologically similar but structurally distinct $\alpha 4 \beta 2$ nicotinic agonist (+)-epibatidine (Damaj et al, 1994), monkeys readily learned to discriminate $0.001 \mathrm{mg} / \mathrm{kg}(+)$-epibatidine from vehicle. These findings suggest that the failure of vaccinated monkeys to acquire nicotine discrimination was due to previous treatment with the nicotine-targeting vaccine SEL-068 and that the protective effects of SEL-068 are highly specific for nicotine. This idea is further supported by observations that treatment with SEL-068 diminished nicotine's, but not (+)-epibatidine's, discriminative-stimulus effects in monkeys that were trained to discriminate nicotine from vehicle prior to vaccination. In this latter group, three of the four subjects failed to reliably identify the previously recognized training dose of $0.1 \mathrm{mg} / \mathrm{kg}$ nicotine for up to 30 weeks following initial treatment with SEL-068. Taken together, our results indicate that immunization with SEL-068: (a) prevented the development of nicotine discrimination in nicotine-naive monkeys; and (b) attenuated the discriminative-stimulus effects of nicotine in nicotineexperienced monkeys. These findings provide the first 
preclinical evidence in a primate species that synthetic nanoparticle-based vaccines that target nicotine can modify stimulus effects of nicotine that may contribute to smoking behavior.

The present results with SEL-068 are consistent with previous findings showing that earlier nicotine-targeting vaccines can effectively: (a) attenuate nicotine's discriminative-stimulus and reinforcing properties; (b) lessen nicotine's withdrawal effects; and (c) influence nicotine's pharmacokinetic properties (eg, Carrera et al, 2004; Lindblom et al, 2002; 2005; LeSage et al, 2006; Raupach et al, 2012; Pentel and LeSage, 2014). However, such promising preclinical evidence has not yet led to the introduction of immunotherapy that can effectively reduce human tobacco consumption, maintain or enhance long-term smoking cessation, or prevent relapse. In clinical trials, only modest increases in quit rates have been observed in smokers with relatively high levels of antinicotine antibodies, and no effect was observed in smokers with relatively low antibody levels (Raupach et al, 2012; Hartmann-Boyce et al, 2012; Pentel and LeSage, 2014). The reasons for this lack of success are uncertain but have been attributed to the absence of a consistently and sufficiently robust immune response (ie, high antibody levels) due to insufficient dosing as a result of study design, safety issues, or variability in response among individual subjects (eg, Raupach et al, 2012; Hartmann-Boyce et al, 2012; Pentel and LeSage, 2014). Phase 1 clinical trials with SEL-068 are currently underway, and at present, it is unknown whether such considerations may also limit the clinical effectiveness of novel nanoparticle-based vaccines for smoking cessation.

Although antibody titers were not obtained in the present experiments, earlier preclinical studies in mice, rhesus monkeys, and cynomolgus monkeys have shown that vaccination with a comparable dose of SEL-068 ( $\geqslant 2 \mathrm{mg})$ every 2 weeks (mice) or 4 weeks (monkeys) consistently produces a strong and selective immune response, evident in the persistent and long-term detection of high-affinity antinicotine antibody titers, particularly after the second and third immunizations (eg, $\alpha$-nicotine IgG $\mathrm{EC}_{50}$ levels $\geqslant 10^{4}$ in both mice and monkeys; Pittet et al, 2012; Kishimoto et al, 2012; Fraser et al, 2014). It is tempting to conclude that such high-affinity antinicotine antibodies following immunization with SEL-068 were responsible for preventing or attenuating nicotine's discriminative-stimulus effects in the present studies. Nevertheless, this interpretation must be viewed cautiously in view of some individual variability in the attenuation of nicotine's effects among vaccinated subjects and in the absence of additional data showing both that SEL-068 can induce a robust immune response in squirrel monkeys and that the level of behavioral response is proportional to the immune response. Moreover, it is yet to be determined whether such promising effects observed with SEL-068 in preclinical studies, eg, high antibody titers across different species (Pittet et al, 2012; Kishimoto et al, 2012; Fraser et al, 2014) and blockade of nicotine's discriminative-stimulus effects in monkeys (present results), can be successfully translated to the clinical setting. Notwithstanding these caveats, novel synthetic nanoparticlebased nicotine-targeting vaccines may offer important advantages over first-generation immunotherapies for smoking cessation. In particular, unlike earlier antinicotine immunotherapies, nanoparticle-based vaccines such as
SEL-068 are self-assembling particles ( $t$ SVP) comprising components that can be modified to perhaps further improve vaccine effectiveness (Pittet et al, 2012; Kishimoto et al, 2012; Fraser et al, 2014). The adjustable features of a nanoparticle vaccine such as SEL-068 are a promising development in nicotine-targeting immunotherapeutic strategies for preventing tobacco consumption (Pentel and LeSage, 2014).

The present results offer some important insights for the continued development of effective nicotine-targeting vaccines. First, multiple immunizations (at least three to four) of SEL-068 were necessary to effectively diminish the effects of nicotine in nicotine-experienced subjects, suggesting that the steady increase in antibody levels eventually achieved a concentration that sufficiently blocked the discriminative-stimulus effects of nicotine. These findings further illustrate the importance of the dose-response relationship in evaluating the effectiveness of vaccine strategies and suggest that, when permissible, clinical study designs should incorporate multiple dosing strategies to achieve antibody titers that may realistically be effective (Hartmann-Boyce et al, 2012). A second concern in evaluating novel vaccines is their safety. Although the present studies were not designed to evaluate the possible adverse effects of SEL-068, it is worth noting that untoward reactions following multiple s.c. injections of the vaccine were not observed in any subject, eg, no changes in body weight, adverse effects at injection site, or changes in overt behavior were observed. Finally, although the prevention of nicotine's discriminative-stimulus effects in laboratory animals may serve as a useful preclinical indicator of a treatment's ability to block nicotine's interoceptive stimulus effects in man, multiple factors govern tobacco addiction (eg, pharmacological properties of multiple tobacco constituents; complex interplay between neurobiological, genetic, environmental, and social factors) and likely influence the overall efficacy of antinicotine vaccines (Pentel and LeSage, 2014). Such considerations do not undermine the view that antinicotine vaccines may be clinically useful, but they do emphasize the complex challenge of reducing nicotine addiction (Pentel and LeSage, 2014).

Perhaps one of the most important features of the current findings is the difference in the effectiveness of SEL-068 against nicotine in subjects that received the vaccine during or after training to discriminate the nicotinic agonist $(+)$-epibatidine and those that were vaccinated before they were trained to discriminate either nicotine or (+)-epibatidine. SEL-068 treatment attenuated, but did not eliminate, the interoceptive effects of nicotine in subjects that were trained to discriminate (+)-epibatidine, whether or not they previously had been trained to discriminate nicotine. This was especially clear in subjects that previously had been trained only with (+)-epibatidine; nicotine failed to substitute fully for the training stimulus even up to doses of nicotine that produced clear evidence of nicotinic physiological effects (eg, emesis following $0.56 \mathrm{mg} / \mathrm{kg}$ ). It is noteworthy that nicotine could not be established as a discriminativestimulus in any of the subjects that underwent the same regimen of vaccination prior to any discrimination training. In contrast, the discriminative-stimulus effects of the full $((+)$-epibatidine) and partial (varenicline) nicotinic agonists were comparable across all subjects in all conditions in which they were tested. These differing effects of nicotine, but not other nicotinic drugs, following comparable regimens of 
SEL-068 treatment suggest that the degree to which SEL-068 treatment attenuated the behavioral effects of nicotine depended on the previous history of exposure: SEL-068 treatment was least effective in subjects that initially were trained to discriminate nicotine from vehicle and most effective in subjects that had not previously learned to discriminate the effects of nicotine. Although higher doses of SEL-068 or a more intensive treatment regimen possibly may have fully blocked nicotine's discriminative-stimulus effects even in subjects that previously had learned to discriminate nicotine fully, these findings illustrate the different degrees of difficulty in preventing the recognition of nicotine's discriminative-stimulus, and perhaps subjective, effects in experienced and naive subjects.

In summary, our results indicate that treatment with the novel nanoparticle-based vaccine SEL-068 selectively prevents the development of nicotine's discriminative-stimulus effects in nicotine-naive monkeys and produces long-term and substantive reductions in the discriminative-stimulus effects of the training dose of nicotine in nicotine-experienced monkeys. These findings provide markedly strong evidence that the ability of SEL-068 to attenuate or block the effects of nicotine in nonhuman primates is highly selective for nicotine among nicotinic agonists. Moreover, the data further suggest that the degree of nicotine inactivation that is necessary for successful treatment with vaccines against nicotine may differ according to clinical end point. Thus, although the merits of vaccination as a preventive measure are extremely controversial, the present results suggest that antinicotine vaccines may be most effective for this purpose in naive individuals or, more cautiously, may be useful in preventing relapse during long-term abstinence in tobacco users. In experienced tobacco users, however, the same vaccination regimen may not be fully effective in preventing nicotine's actions and the possibility that more limited effects may lead to increased smoking as compensatory behavior, while previously discounted, (Raupach et al, 2012; Hartmann-Boyce et al, 2012; Pentel and LeSage, 2014) still must be considered in evaluating the clinical efficacy of this strategy. Possibly, a combination treatment strategy, ie, nanoparticle-based nicotine vaccine together with a nicotinic partial agonist such as varenicline or antagonist such as mecamylamine, might more effectively prevent the addiction-related effects of nicotine among experienced tobacco users. Such a view is bolstered by a recent study which showed that mecamylamine combined with an earlier nicotine-targeting vaccine can: (a) synergistically attenuate the discriminative-stimulus effects of nicotine in rats and (b) enhance the efficacy of nicotine vaccines in preventing nicotine's stimulus properties (LeSage et al, 2012; Pentel and LeSage, 2014). The extent to which this approach may be more effective than currently available pharmacotherapies in promoting smoking cessation among experienced smokers is important and deserves further attention.

\section{FUNDING AND DISCLOSURE}

The authors declare that, except for income received from our primary employer, no other financial support or compensation has been received from any individual or corporate entity over the past three years for research or for professional services, and there are no personal financial holdings that could be perceived as constituting a potential conflict of interest. Selecta Biosciences (Watertown, MA) provided funds to support this research. The authors declare no conflict of interest.

\section{ACKNOWLEDGMENTS}

We thank Dr Hans Rollema (Pfizer) and the NIDA Drug Supply Program for providing, respectively, varenicline and (+)-epibatidine for the present studies, and Dr BD Kangas and Dr CA Paronis for their comments on an earlier version of this manuscript. We also thank Selecta Biosciences (Watertown, MA) for providing SEL-068.

\section{AUTHOR CONTRIBUTIONS}

Deasi and Bergman participated in research design for behavioral experiments, performed behavioral data analysis, and contributed to the writing of the manuscript. Desai conducted behavioral experiments and acquired funding for the research.

\section{REFERENCES}

Carrera MR, Ashley JA, Hoffman TZ, Isomura S, Wirsching P, Koob GF et al (2004). Investigations using immunization to attenuate the psychoactive effects of nicotine. Bioorg Med Chem 12: $563-570$.

Cunningham CS, Javors M, McMahon LR (2012). Pharmacologic characterization of a nicotine discriminative stimulus in rhesus monkeys. J Pharmacol Exp Ther 341: 840-849.

Damaj MI, Creasy KR, Grove AD, Rosecrans JA, Martin BR (1994). Pharmacological effects of epibatidine optical enantiomers. Brain Res 664: 34-40.

Desai RI, Bergman J (2014). Methamphetamine-like discriminativestimulus effects of nicotinic agonists. J Pharmacol Exp Ther 348: 478-488.

Desai RI, Barber DJ, Terry P (2003). Dopaminergic and cholinergic involvement in the discriminative-stimulus effects of nicotine and cocaine in rats. Psychopharmacology (Berl) 167: 335-343.

de Villiers SH, Lindblom N, Kalayanov G, Gordon S, Malmerfelt A, Johansson AM et al (2002). Active immunization against nicotine suppresses nicotine-induced dopamine release in the rat nucleus accumbens shell. Respiration 69: 247-253.

Fraser CC, Altreuter DH, Ilyinskii P, Pittet L, LaMothe RA, Keegan $M$ et al (2014). Generation of universal CD4 memory $\mathrm{T}$ cell recall peptide effective in humans and non-human primates. Vaccine 32: 2896-2903.

Hartmann-Boyce J, Cahill K, Hatsukami D, Cornuz J (2012). Nicotine vaccines for smoking cessation. Cochrane Datebase Syst Rev 8: CD007072.

Jutkiewicz EM, Brooks EA, Kynaston AD, Rice KC, Woods JH (2011). Patterns of nicotinic receptor antagonism: nicotine discrimination studies. J Pharmacol Exp Ther 339: 194-202.

Kamien JB, Bickel WK, Hughes JR, Higgins ST, Smith BJ (1993). Drug discrimination by humans compared to nonhumans: current status and future directions. Psychopharmacology (Berl) 111: 259-270.

Kelleher RT, Morse WH (1968). Determinants of the specificity of behavioral effects of drugs. Ergebnisse Physiol 60: 1-56.

Kishimoto K, Altreuter D, Johnston L, Keller P, Pittet L (2012). SEL-068 A fully synthetic nanoparticle vaccine for smoking cessation and relapse prevention. SRNT 2012 Annual Meeting: Houston, TX, USA.

LeSage MG, Shelley D, Pravetoni M, Pentel PR (2012). Enhanced attenuation of nicotine discrimination in rats by combining 
nicotine-specific antibodies with a nicotinic receptor antagonists. Pharmacol Biochem Behav 102: 157-162.

LeSage MG, Keyler DE, Hieda Y, Collins G, Burroughs D, Le C et al (2006). Effects of nicotine conjugate vaccine on the acquisition and maintenance of nicotine self-administration in rats. Psychopharmacology (Berl) 184: 409-416.

Lindblom N, de Villers SH, Kalayanov G, Gordon S, Johansson AM, Svensson TH (2002). Active immunization against nicotine prevents reinstatement of nicotine-seeking behavior in rats. Respiration 69: 254-260.

Lindblom N, de Villers SH, Semenova S, Kalayanov G, Gordon S, Schilstrom B et al (2005). Active immunisation against nicotine blocks the reward facilitating effects of nicotine and partially prevents nicotine withdrawal in the rat as measured by dopamine output in the nucleus accumbens, brain reward thresholds and somatic signs. Naunyn Schmiedebergs Arch Pharmacol 372: 182-194.

Pentel PR, LeSage MG (2014). New directions in nicotine vaccine design and use. Adv Pharmacol 69: 553-580.

Perkins KA (2009). Discriminative stimulus effects of nicotine in humans. Handb Exp Pharmacol 192: 369-400.

Pittet L, Altreuter DH, IIyinskii P, Fraser CC, Gao Y, Baldwin S et al (2012). Development and preclinical evaluation of SEL-068, a novel targeted Synthetic Vaccine Particle $\left(t \mathrm{SVP}^{\mathrm{TM}}\right)$ for smoking cessation and relapse prevention that generates high titers of antibodies against nicotine. J Immunol 188 (75.11).

Polosa R, Benowitz NL (2011). Treatment of nicotine addiction: present therapeutic options and pipeline developments. Trends Pharmacol Sci 32: 281-290.

Raupach T, Hoogsteder PH, Onnovan Schayck CP (2012). Nicotine vaccines to assist with smoking cessation: current status of research. Drugs 72: e1-e16.

Smith JW, Stolerman IP (2009). Recognising nicotine: the neurobiological basis of nicotine discrimination. Handb Exp Pharmacol 192: 295-333.

Tidey JW, Bergman J (1998). Drug discrimination in methamphetamine-trained monkeys: agonist and antagonist effects of dopaminergic drugs. J Pharmacol Exp Ther 285: 1163-1174.

US Department of Health and Human Services (USDHHS) The Health Consequences of Smoking - 50 years of Progress: A Report of the Surgeon General. U.S. Department of Health and Human Services, Centers for Disease Control and Prevention, National Center for Chronic Disease Prevention and Health Promotion, Office on Smoking and Health, USA, 2014. 